\title{
Acellular dermal matrix (Insuregraf) in the prevention of Frey's syndrome and surgical site depression after parotidectomy
}

Jangyoun Choi ${ }^{1}$,

Song I Park ${ }^{1}$,

Eun Young Rha ${ }^{2}$,

Bommie Florence Seo ${ }^{1}$,

Ho Kwon ${ }^{1}$,

Sung-No Jung ${ }^{1}$

${ }^{1}$ Department of Plastic and

Reconstructive Surgery, Uijeongbu St. Mary's Hospital, College of Medicine,

The Catholic University of Korea,

Uijeongbu; ${ }^{2}$ Department of Plastic and Reconstructive Surgery, Eunpyeong St. Mary's Hospital, College of Medicine,

The Catholic University of Korea, Seoul, Korea
Background: Parotidectomy is the treatment of choice in many parotid tumors. Due to the extensive nature of the procedure, unfavorable complications such as gustatory sweating, surgical site depression are common. Various techniques using fascia, muscle or AlloDerm have been developed but debate still remains regarding its availability and affordability. We applied a newly developed acellular dermal matrix (Insuregraf) to the parotidectomy field to act as a physical barrier and to provide adequate filling effect for prevention of functional and aesthetic complications.

Methods: From March 2010 to March 2017, 30 patients with parotid tumors underwent superficial parotidectomy. Twenty patients underwent only superficial parotidectomy. Ten patients had Insuregraf applied to the surgical site after superficial parotidectomy. We evaluated the incidence of Frey's syndrome, surgical site depression, and patient satisfaction rate in both groups.

Results: The incidence of Frey's syndrome was lower in the Insuregraf group (0 vs. 2). Surgical site depression was also lower in the Insuregraf group (2 vs. 20). Satisfaction score for facial contour in Insuregraf group was 9.2 out of 10 , which was comparable to 6.2 out of 10 in the control group.

Conclusion: Application of Insuregraf after superficial parotidectomy is an effective surgical procedure to prevent complications such as Frey's syndrome and surgical site depression. This technique is affordable and safe with no immune reactions. Above all this surgical method should be considered as an option for patients who are concerned about the contour of the face after surgery.

Keywords: Acellular dermis / Collagen / Facial asymmetry / Parotid neoplasms / Sweating, gustatory

\section{INTRODUCTION}

The parotid tumors account for about $3 \%$ of the head and neck tumors and account for $80 \%$ of the tumors in the salivary gland $[1,2]$. Due to its benign but extensive nature, it is usually treated

\footnotetext{
Correspondence: Sung-No Jung

Department of Plastic and Reconstructive Surgery, Uijeongbu St. Mary's Hospital, College of Medicine, The Catholic University of Korea, 271 Cheonbo-ro, Uijeongbu 11765 , Korea

E-mail: jsn7190@hanmail.net

Received May 22, 2019 / Revised May 31, 2019 / Accepted June 7, 2019
}

by removing the parotid gland. Due to the encasement of the facial nerve inside the parotid gland, prevention of the facial nerve was the most important focus during the surgical procedure. It is a fact that other complications such as Frey's syndrome, surgical site depression, ear sensory abnormality, scar, pain, and dry mouth have been relatively overlooked [3]. The most common complication is Frey's syndrome and depression of the surgical site [4]. Frey's syndrome is also known as gustatory sweating or auriculotemporal nerve syndrome. It is characterized by sweating and flushing of the parotid area skin due 
to the aberrant nerve connections made between nerves of the skin and the remaining parotid gland [5]. Since the first report of Duphenix in 1757, many publications have reported an incidence of $11 \%$ to $95 \%$ [6,7]. In 1923, Frey [5] reported that the occurrence of Frey's syndrome was associated with auriculotemporal nerve. In 1927, Andre Thomas suggested that parasympathetic nerves grow abnormally in the sweat glands and blood vessels of the skin covering the parotid gland, causing Frey's syndrome. Depression of the surgical site after parotidectomy results in facial asymmetry and occurs in most patients $[4,8]$. Frey's syndrome and skin depression after parotidectomy are very common and cause significant aesthetic and quality of life issues. To prevent such complications, many surgical methods have been introduced to prevent abnormal nerve connection. This is usually achieved by placing a physical barrier such as fascia, muscle or artificial dermal matrix between the parotid gland and the skin. Most widely reported method is the placement of AlloDerm sheet over the dissected plane. However, its accessibility is limited due to high cost and limited amount of source from cadaveric skin.

Insuregraf (SK Bioland, Cheonan, Korea) is a highly-porous, native dermal matrix consisting of $100 \%$ of type I collagen derived from porcine skin which can provide a scaffold structure for an organized colonization by dermal cells and facilitate the reconstruction of soft tissue defect. There have been a few reports on utilizing Insuregraf in burn victims, which showed excellent results in terms of dermal layer organization, skin thickness, and skin elasticity. In this regard, we hypothesized that Insuregraf could simultaneously act as a physical barrier and provide adequate tissue thickness after parotidectomy. In this article, we report our experience of utilizing Insuregraf as an alternative material for prevention of common complications following superficial parotidectomy.
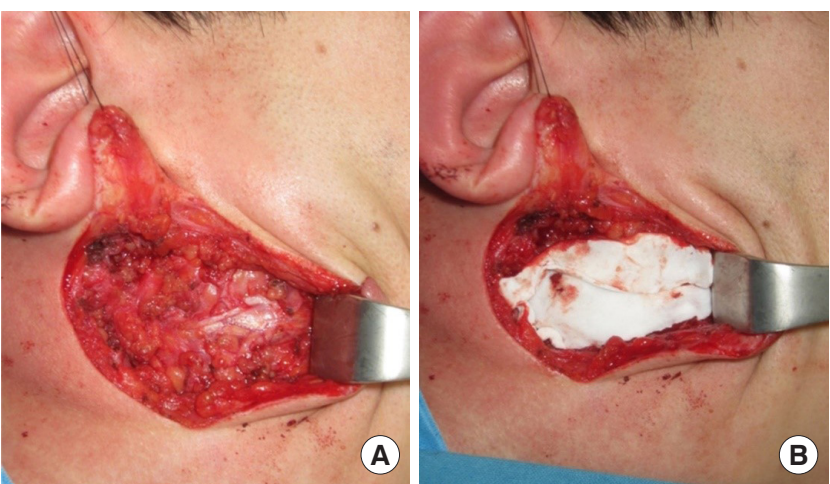

Fig. 1. A 21-year-old man with a pleomorphic adenoma on his right parotid gland area for 6 months. (A) After superficial parotidectomy. (B) Insertion of Insuregraf after parotidectomy.

\section{METHODS}

\section{Study design and patient demographics}

This study was approved by the Institutional Review Board of Catholic University of Korea (IRB No. UC18RESI0063). This study included 30 patients who underwent superficial parotidectomy from March 2010 to March 2017. The patients were then randomly divided into two groups: 20 patients (control group) underwent superficial parotidectomy only and 10 patients (Insuregraf group) received superficial parotidectomy and Insuregraf was placed before skin closure.

\section{Surgical technique}

All operations were performed by a single surgeon (SNJ) and the operative procedure was uniform in all cases of this study, which consisted of superficial parotidectomy. A lazy-S shaped Blair incision was applied from the preauricular area to the proximal one-third of mandibular body. Skin flap was elevated to the anterior margin of the masseter muscle. In the control group, skin flap was directly closed after the excision of the superficial parotid gland. For the Insuregraf group, a matrix sheet of Insuregraf was placed over the superficial parotidectomy field (Fig. 1). Insuregraf was trimmed to fit the depressed area and was $4 \times 8 \mathrm{~cm}$ in size on average. Insuregraf was fixed to the surrounding tissues using Tisseel (Baxter, Deerfield, IL, USA) for greater stability and the skin flap was closed (Fig. 2). A small sized negative suction drain was placed inside the surgical field in both groups for 3 to 7 days for prevention of hematoma.

\section{Evaluation of surgical outcome}

The symptoms of Frey's syndrome and facial asymmetry due to surgical site depression were evaluated at 18 months postoperatively. A different physician uninvolved in the surgery was in-
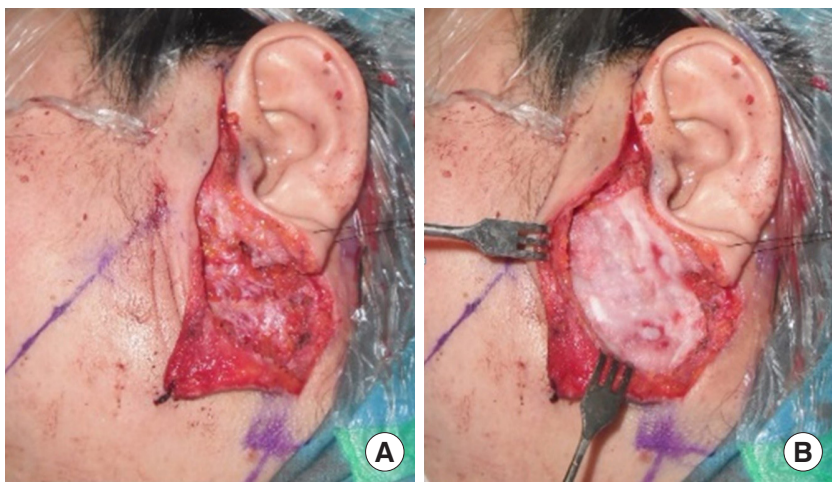

Fig. 2. A 51-year-old woman with a Warthin tumor on her left parotid area found accidentally after a computed tomography scan. (A) After superficial parotidectomy. (B) Insertion of Insuregraf and fixation to surrounding tissue with Tisseel after parotidectomy. 
Table 1. Demographics and pathologic summary of the patients enrolled in the study

\begin{tabular}{lcc} 
& Control group & Insuregraf group \\
\hline No. of patients & 20 & 10 \\
Sex & 15 & 3 \\
$\quad$ Male & 5 & 7 \\
$\quad$ Female & $36.6(25-57)$ & $37.7(21-60)$ \\
Age (yr) & & \\
Pathologic type & 17 & 9 \\
Pleomorphic adenoma & 2 & 1 \\
Warthin tumor & 1 & 0 \\
Schwannoma & & \\
\hline
\end{tabular}

Values are presented as number or mean (range).

vited to evaluate the presence of surgical site depression and Frey's syndrome. Patient overall satisfaction for facial contour was evaluated with a 10-point scale questionnaire.

\section{Statistical analysis}

Incidence of Frey's syndrome and surgical site depression was compared with Fisher exact test. The mean satisfaction score was analyzed with Mann-Whitney test.

\section{RESULTS}

Of the 30 patients, male to female ratio was 18 to 12 (60\% vs. $40 \%)$. The mean age was 37 years (range, 21-60 years). Of these, 26 patients had pleomorphic adenoma, three patients had Warthin tumor and one patient was diagnosed with schwannoma (Table 1).

All 30 patients completed the study. No cases of facial nerve damage, immune reaction to the graft material, or salivary fistula was observed in either group. Frey's syndrome did not occur $(0 \%)$ in the Insuregraf group. Two patients (10\%) experienced Frey's syndrome in the control group (Fisher exact test, $p=0.54$ ). Surgical site depression occurred in two patients (20\%) in the Insuregraf group and 20 patients $(100 \%)$ in the control group (Fisher exact test, $p<0.01$ ). The mean satisfaction score for facial contours was 9.2 out of 10 in the Insuregraf group and 6.2 out of 10 in the control group, showing a significantly higher score in the Insuregraf group (Mann-Whitney test, $p<0.01$ ) (Table 2).

\section{DISCUSSION}

Preventing Frey's syndrome and surgical site depression after parotidectomy procedure has been a longtime concern. Various methods have been reported to prevent complications after pa-
Table 2. Postoperative results

\begin{tabular}{lccc}
\hline & Control group & Insuregraf group & $p$-value \\
\hline Frey's syndrome & $2(10)$ & 0 & 0.54 \\
Surgical site depression & $20(100)$ & $2(20)$ & $<0.01^{\text {a) }}$ \\
Mean satisfaction score & 6.2 & 9.2 & $<0.01^{\text {a) }}$ \\
\hline
\end{tabular}

Values are presented as number (\%).

a) Significant differences are indicated by $p<0.01$.

rotidectomy. Autogenous vascularized flaps such as the sternocleidomastoid (SCM) muscle flap, the superficial musculoaponeurotic system (SMAS) flap, the temporoparietal flap, and the platysma flap are the procedures that have been used before artificial dermal matrix products gained popularity. Although widely supported, each strategy retains its own drawbacks. SCM muscle flap can provide bulk and is suitable for cases where severe depression is expected. However, possible injury to the spinal accessory nerve, and delayed detection of tumor recurrence under the thick muscle bulk are major drawbacks [9-11]. Temporoparietal fascia is a versatile flap arising from the superficial temporal artery. This flap is wide enough to completely cover the exposed parotid gland and facial nerve. However, additional scalp incision followed by scar alopecia, hollowness of the temporal area is a major limitation of this technique [12-14]. The SMAS flap, the most widely used, can be used as superior rotation, posterior advancement or plication as well as redraping method depending on the location and size of the defect [15]. Surgical procedure is relatively simple because the flap can be elevated in the same surgical field [16]. However, it is impossible to employ this technique for extensive tumors, which sometimes requires en bloc removal of the tumor including the SMAS tissue [17]. SMAS flap is thinner than SCM muscle flap and is not suitable for large defect sites [13-17].

The use of autogenous non-vascularized tissues such as fascia lata, free fat or dermofat has been attempted for decades to cover the defect site after parotidectomy. However, no large-scale studies using this method have been published.

The fat graft has a similar texture to the parotid gland, which is a good way to supplement the defect after parotidectomy [18]. Moreover, this method is easy to handle and the donor site scar is small [18-20]. However, the rate of absorption varies from $25 \%$ up to $80 \%$, and complications such as fat liquefaction, seroma and sialocele may occur [21-23]. To overcome the problem of reabsorption after fat graft, transplantation with dermis or overcorrected $15 \%$ to $30 \%$ has been used $[13,19,20]$. However, dermal fat grafts also vary in absorption rate and can cause inflammation due to dregs of dermis [19].

Recently, various allogenic implants have been introduced to fill the parotid defect [8,21-25]. These methods are easy, have a 
Table 3. Efficacy of ADM for parotidectomy defects presented in previous studies

\begin{tabular}{|c|c|c|c|c|c|c|c|}
\hline Author & Year & Country & Group & No. & $\begin{array}{l}\text { Follow-up } \\
\text { (mo) }\end{array}$ & $\begin{array}{c}\text { Frey's syndrome, } \\
\text { no. (\%) }\end{array}$ & $\begin{array}{l}\text { Facial contour deformity, } \\
\text { no }(\%)\end{array}$ \\
\hline \multirow[t]{2}{*}{ Govindaraj et al. [24] } & 2001 & USA & AlloDerm & 32 & 6 & $1(3.1)$ & - \\
\hline & & & Control & 32 & & $3(9.3)$ & \\
\hline \multirow[t]{2}{*}{ Sinha et al. [25] } & 2003 & USA & AlloDerm & 10 & 12 & $1(10.0)$ & - \\
\hline & & & Control & 10 & & $5(50.0)$ & \\
\hline \multirow[t]{2}{*}{ Ye et al. [22] } & 2008 & China & Renov ADM & 64 & 16 & $1(1.5)$ & - \\
\hline & & & Control & 104 & & $63(60.6)$ & \\
\hline \multirow[t]{2}{*}{ Luo et al. [8] } & 2012 & China & J1-ADM & 29 & 6 & $1(3.4)$ & 0 \\
\hline & & & Control & 41 & & $14(34.1)$ & $41(100)$ \\
\hline
\end{tabular}

ADM, acellular dermal matrix.

short operation time, and do not cause donor problems $[8,25]$. AlloDerm is a commonly used acellular dermal matrix (ADM) obtained from cadaveric skin and it has been used increasingly in the last decade. Previous studies have reported that ADM materials, including AlloDerm, are effective in lowering the incidence of Frey's syndrome and bringing good cosmetic results (Table 3). Despite its excellent characteristics, the material is very expensive and its supply is limited. Furthermore, it requires refrigeration for storage and requires over 30 minutes of hydration before implantation.

Insuregraf, the material we incorporated in this study, is a three-dimensional matrix composed of porcine collagen, which shows high similarity to human dermis. In contrast with AlloDerm, Insuregraf is sufficient in its supply due to abundance of porcine dermis material. Furthermore, it does not require prehydration. The native structurally intact collagen serves as an essential component of the new extracellular matrix for the migration of cells and vascularization. The programmed thickness of Insuregraf allows initial supply to the graft by diffusion and rapid vascularization. According to our results, use of Insuregraf exhibited excellent surgical outcomes compared to the control group, in terms of surgical site depression, incidence of Frey's syndrome, and patient satisfaction. However, difference in the prevalence of Frey's syndrome failed to show any significant results. However, considering the structural property and chemical composition of Insuregraf, we assume that it has sufficient potential ability to yield significant differences in a large group study. Therefore, we think that Insuregraf can be a feasible choice for preventing common complications after parotidectomy. Compared to other methods, this material shows advantage in its convenience in storage, direct usage, and low cost.

Due to our small sample size and limited parameters for evaluation, further studies with larger sample scale are definitely needed. A head-to-head comparison study with AlloDerm can be undertaken for future investigation.
Insuregraf, a porcine dermal matrix sheet, improved surgical outcomes compared with control. It reduced the occurrence of Frey's syndrome and surgical site depression. Patient overall satisfaction scores for postsurgical cosmesis were higher. We think that Insuregraf can be an alternative method to autologous reconstruction in terms of shorter surgical time and low surgical morbidity. Compared to AlloDerm, Insuregraf is easily affordable, and much more convenient to use in terms of storage and implantation. Therefore, we believe that Insuregraf can be an efficient option for better functional and esthetic outcomes after parotidectomy procedures.

\section{NOTES}

\section{Conflict of interest}

No potential conflict of interest relevant to this article was reported.

\section{Ethical approval}

The study was approved by the Institutional Review Board of the Catholic University of Korea (IRB No. UC18RESI0063) and performed in accordance with the principles of the Declaration of Helsinki. Written informed consents were obtained.

\section{Patient consent}

The patients provided written informed consent for the publication and the use of their images.

\section{ORCID}

Jangyoun Choi https://orcid.org/0000-0002-5165-8414 Song I Park https://orcid.org/0000-0002-9536-6376

Eun Young Rha https://orcid.org/0000-0001-9435-4026

Bommie Florence Seo https://orcid.org/0000-0002-6907-5962

Ho Kwon https://orcid.org/0000-0001-7471-0804

Sung-No Jung https://orcid.org/0000-0002-0419-4717 


\section{REFERENCES}

1. Donati M, Gandolfo L, Privitera A, Brancato G, Cardi F, Donati A. Superficial parotidectomy as first choice for parotid tumours. Chir Ital 2007;59:91-7.

2. Skolnik EM, Friedman M, Becker S, Sisson GA, Keyes GA. Tumors of the major salivary glands. Laryngoscope 1977;87:84361.

3. Nitzan D, Kronenberg J, Horowitz Z, Wolf M, Bedrin L, Chaushu G, et al. Quality of life following parotidectomy for malignant and benign disease. Plast Reconstr Surg 2004;114: 1060-7.

4. Liu DY, Tian XJ, Li C, Sun SS, Xiong YH, Zeng XT. The sternocleidomastoid muscle flap for the prevention of Frey syndrome and cosmetic deformity following parotidectomy: a systematic review and meta-analysis. Oncol Lett 2013;5:1335-42.

5. Frey L. Le syndrome du nerf auriculo-temporal. Rev Neurol 1923;2:97-104.

6. de Bree R, van der Waal I, Leemans CR. Management of Frey syndrome. Head Neck 2007;29:773-8.

7. Koch M, Zenk J, Iro H. Long-term results of morbidity after parotid gland surgery in benign disease. Laryngoscope 2010; 120:724-30.

8. Luo W, Zheng X, Chen L, Jing W, Tang W, Long J, et al. The use of human acellular dermal matrix in the prevention of infraauricular depressed deformities and Frey's syndrome following total parotidectomy. Oral Surg Oral Med Oral Pathol Oral Radiol 2012;114:e9-13.

9. Casler JD, Conley J. Sternocleidomastoid muscle transfer and superficial musculoaponeurotic system plication in the prevention of Frey's syndrome. Laryngoscope 1991;101(1 Pt 1):95-100.

10. Sood S, Quraishi MS, Jennings CR, Bradley PJ. Frey's syndrome following parotidectomy: prevention using a rotation sternocleidomastoid muscle flap. Clin Otolaryngol Allied Sci 1999;24:365-8.

11. Gooden EA, Gullane PJ, Irish J, Katz M, Carroll C. Role of the sternocleidomastoid muscle flap preventing Frey's syndrome and maintaining facial contour following superficial parotidectomy. J Otolaryngol 2001;30:98-101.

12. Cesteleyn L, Helman J, King S, Van de Vyvere G. Temporoparietal fascia flaps and superficial musculoaponeurotic system plication in parotid surgery reduces Frey's syndrome. J Oral Maxillofac Surg 2002;60:1284-97.
13. Sultan MR, Wider TM, Hugo NE. Frey's syndrome: prevention with temporoparietal fascial flap interposition. Ann Plast Surg 1995;34:292-6.

14. Ahmed OA, Kolhe PS. Prevention of Frey's syndrome and volume deficit after parotidectomy using the superficial temporal artery fascial flap. Br J Plast Surg 1999;52:256-60.

15. Moulton-Barrett R, Allison G, Rappaport I. Variation's in the use of SMAS (superficial musculoaponeurotic system) to prevent Frey's syndrome after parotidectomy. Int Surg 1996;81: 174-6.

16. Curry JM, Fisher KW, Heffelfinger RN, Rosen MR, Keane WM, Pribitkin EA. Superficial musculoaponeurotic system elevation and fat graft reconstruction after superficial parotidectomy. Laryngoscope 2008;118:210-5.

17. Honig JF. Facelift approach with a hybrid SMAS rotation advancement flap in parotidectomy for prevention of scars and contour deficiency affecting the neck and sweat secretion of the cheek. J Craniofac Surg 2004;15:797-803.

18. Chan LS, Barakate MS, Havas TE. Free fat grafting in superficial parotid surgery to prevent Frey's syndrome and improve aesthetic outcome. J Laryngol Otol 2014;128 Suppl 1:S44-9.

19. Nosan DK, Ochi JW, Davidson TM. Preservation of facial contour during parotidectomy. Otolaryngol Head Neck Surg 1991; 104:293-8

20. Harada T, Inoue T, Harashina T, Hatoko M, Ueda K. Dermisfat graft after parotidectomy to prevent Frey's syndrome and the concave deformity. Ann Plast Surg 1993;31:450-2.

21. Sachsman SM, Rice DH. Use of AlloDerm implant to improve cosmesis after parotidectomy. Ear Nose Throat J 2007;86:512-3.

22. Ye WM, Zhu HG, Zheng JW, Wang XD, Zhao W, Zhong LP, et al. Use of allogenic acellular dermal matrix in prevention of Frey's syndrome after parotidectomy. Br J Oral Maxillofac Surg 2008;46:649-52.

23. Athavale SM, Phillips S, Mangus B, Datta J, Sinard RJ, Netterville JL, et al. Complications of AlloDerm and dermamatrix for parotidectomy reconstruction. Head Neck 2012;34:88-93.

24. Govindaraj S, Cohen M, Genden EM, Costantino PD, Urken ML. The use of acellular dermis in the prevention of Frey's syndrome. Laryngoscope 2001;111(11 Pt 1):1993-8.

25. Sinha UK, Saadat D, Doherty CM, Rice DH. Use of AlloDerm implant to prevent Frey syndrome after parotidectomy. Arch Facial Plast Surg 2003;5:109-12. 